\title{
Biological Agent Type
}

National Cancer Institute

\section{Source}

National Cancer Institute. Biological Agent Type. NCI Thesaurus. Code C158308.

The type of biological agent employed in the model. 